\title{
FACTORES BIOPSICOSOCIALES QUE SE RELACIONAN CON EL FUNCIONAMIENTO DE LAS FAMILIAS CON PACIENTES DEPENDIENTES
}

\author{
BIOPSYCHOSOCIAL FACTORS RELATED TO FUNCTIONING \\ OF FAMILIES WITH DEPENDANT PATIENTS
}

\author{
Romina Gabriela Moraga Jaramillo* \\ Patricia Roxena Cid Henríquez ${ }^{* *}$
}

\begin{abstract}
RESUMEN
Objetivo: Identificar cómo se relacionan los factores biopsicosociales, factores de riesgo y protectores con el funcionamiento familiar de las familias que tienen un integrante dependiente, desde el punto de vista del paciente y la familia. Material y método: Estudio transversal, descriptivo, correlacional. La población correspondió a 154 pacientes y 310 familiares que vivían con un integrante dependiente adscritos a centros de salud familiar de la ciudad de Chilán, Chile. El instrumento recolector de datos estuvo conformado por un cuestionario semiestructurado que midió variables biopsicosociales y las escalas FACES III y de Comunicación incluida en FACES IV para evaluar las variables de Cohesión, Adaptabilidad y Comunicación. Resultados: De los 16 tipos de familias que propone Olson, al combinar los cuatro tipos de Cohesión y cuatro tipos de Adaptabilidad del familiar, según la percepción de los familiares encuestados, sólo se encontraron 12 tipos de ellos. El 24,5\% tuvo un funcionamiento familiar separado-caótico, seguido del 24,0\% conectado-caótico, es decir rango medio. Un 23,9\% es desligada-caótica, es decir funcionamiento familiar extremo, presentando alteración en el funcionamiento familiar. Se encontró una asociación estadística significativa entre los tipos de comunicación, percibida por el familiar y paciente, y el funcionamiento familiar. Conclusión: Las familias que viven con un paciente dependiente presentan alteración en el Funcionamiento familiar y la Comunicación es un elemento que favorece la dinámica familiar. Esta información contribuye a entregar un cuidado de enfermería acorde con las necesidades específicas de estas familias en el contexto del Modelo de Salud Familiar.
\end{abstract}

Palabras claves: Relaciones familiares, adaptación, comunicación, cuidadores, enfermería de la familia.

\begin{abstract}
Objective: The aim of this study was to identify how biopsychosocial factors, risk factors and protectors are related to family functioning within the dimensions of cohesion and adaptability on those families which have a dependent member attached to Family Health-Care Centers. Method: Cross-sectional, descriptive and correlational study. The population corresponded to 154 patients and 310 families that lived with a dependent member attached to family health centers in the city of Chillán. Data collection was done through a semistructured survey, FACES III scales and a communication scale included in FACES IV in order to measure the research variables. Results: Out of the 16 types of families proposed by Olson, when combining the four types of Cohesion and four types of Adaptability of the family, according to the perception of the relatives surveyed, only 12 types were found in the families studied. $24.5 \%$ had separate, chaotic family functioning, followed by $24.0 \%$ who displayed connected-chaotic functioning, that is, middle range. $23.9 \%$ was classified as disconnected-

\footnotetext{
*Enfermera. Docente Departamento de Enfermería Universidad del Bío-Bío, Chillán Chile. Email: romimoraga@gmail.com

${ }^{* *}$ Enfermera. Docente Facultad de Enfermería Universidad de Concepción, Concepción Chile. Email: patcid@udec.cl
} 
chaotic i.e. extreme family functioning, presenting alteration in family functioning. Conclusion: The results lead to the conclusion that families with dependent patients show a variation within family functioning and communication becomes a vital element which promotes good family dynamic. This information contributes to delivering nursing care wich is consistent with families' specific needs, according to the Family Health-Care model context.

Key words: Family relationships, adaptation, communication, caregivers, family nursing.

Fecha recepción: 12/12/14 Fecha aceptación: 28/12/15

\section{INTRODUCCIÓN}

En Chile, los índices de discapacidad han impactado nuestra realidad. Es así como el $12,9 \%$ de la población chilena vive con alguna discapacidad, 1 de cada 8 chilenos tiene discapacidad y el 2,5\% tiene una discapacidad severa (1). Se estima que estas cifras aumentarán a medida que se incrementen los años de vida de las personas, existiendo un riesgo de presentar enfermedades degenerativas cardiovasculares, osteoarticulares y de salud mental, con una carga considerable a nivel familiar (2). Indudablemente, cuando un integrante de la familia es diagnosticado con algún grado de dependencia o postración, experimenta estrés y debe adaptarse a este evento no normativo y de gran impacto, atribuyéndose la responsabilidad del cuidado, generalmente, a un integrante de la familia, correspondiendo en gran medida a la dueña de casa $(3,4)$.

Frente a esta problemática cada vez mayor, el Ministerio de Salud chileno desde el año 1998 ha creado programas con énfasis en intervenciones de la salud en las personas y sus familias como unidad de cuidados (5). Este enfoque se refuerza con el Modelo de Atención Integral con Enfoque Familiar y Comunitario, el cual fue legalmente aprobado el año 2001 (6). Bajo esta mirada, la salud es responsabilidad de la persona, de su familia y también de la comunidad, siendo fuente de salud o enfermedad para cada uno de sus miembros (7).
Por su parte, el profesional de Enfermería, en su rol de Gestor del Cuidado (8), responde a este Modelo a través del liderazgo en el Programa de Atención Domiciliaria a Personas con Dependencia Severa. Este programa permite reforzar el cuidado tanto a la persona y a las familias, proporcionando herramientas para la valoración, diagnóstico e intervención en la salud de ellas. Para ello, es fundamental valorar el funcionamiento familiar y definir el nivel de dependencia y condición biopsicosocial a través de pautas que identifican factores protectores y de riesgo (9). Esto permite desarrollar cuidados específicos según el funcionamiento de las familias.

Respecto a la comprensión de la familia como sistema, a finales de los años setenta, los estudios iniciados por Olson trataban de mostrar los aspectos más relevantes del funcionamiento familiar, siendo la cohesión y adaptabilidad los principales elementos de este constructo (10) y la comunicación un tercer elemento facilitador de la dinámica familiar. Posteriormente, Olson et al. desarrollaron en 1979 el Modelo Circumplejo, siendo uno de los más utilizados para evaluar el funcionamiento familiar (11). Con base en este modelo se desarrolló un instrumento llamado Family Adaptability and Cohesion Scales (FACES III), que mide dos dimensiones: cohesión y adaptabilidad. Se hipotetiza que ambas dimensiones se relacionan en forma curvilínea con el funcionamiento familiar $(12,13)$. El Modelo Circumplejo permite identificar 16 tipos de sistemas familiares, dividiendo cada dimensión en cuatro nive- 
les: cohesión (desligada, separada, conectada, enmarañada) y adaptabilidad (caótica, flexible, estructurada y rígida). La dimensión adaptabilidad es definida como la calidad y expresión del liderazgo, organización, roles, reglas y negociaciones existentes en la familia. Sus indicadores específicos incluyen: liderazgo, asertividad, control, disciplina, estilos de negociación, relaciones de rol y reglas relacionales. La dimensión cohesión es definida como los lazos emocionales que los miembros de la familia tienen entre sí. Sus indicadores específicos incluyen: cercanía emocional, límites, fronteras, coaliciones, tiempo, espacio, amistades, toma de decisiones, intereses y recreación. La dimensión comunicación se considera un facilitador del Modelo y no es medida en Faces III. Los 16 tipos pueden ser agrupados en tres tipos más generales: balanceadas, intermedias y extremas. Se puede hipotetizar que las familias con cohesión y adaptabilidad equilibradas (dos niveles centrales), por lo general funcionarán de manera más adecuada a lo largo del ciclo vital que aquellas que ocupan los extremos de estas dimensiones. De esta manera, identificar el tipo de funcionamiento familiar se transforma en una valiosa orientación y herramienta para el trabajo terapéutico (14).

Cabe señalar que el funcionamiento familiar saludable posibilita a la familia cumplir exitosamente con las funciones que le están histórica y socialmente asignadas, para lo cual se hace necesario el desarrollo de una dinámica familiar apropiada (15). Esto se refiere al establecimiento de determinadas pautas de interrelación entre sus miembros, las que se encuentran mediadas o matizadas por la expresión de sentimientos, afectos y emociones entre sí, y en relación con el grupo en su conjunto (16). Esto permite a la familia enfrentar adecuadamente las situaciones de crisis, manteniendo la cohesión y adaptabilidad familiar $(17,18)$.

Las investigaciones sobre funcionamiento familiar se han llevado a cabo para evaluar las relaciones familiares de pacientes y familiares con distintas variables biopsicosociales y de enfermedades, en distintos grupos etáreos $(12,19,20)$. Entre los estudios realizados sobre dinámica y funcionamiento de familias de atención primaria de salud, se encuentra el efectuado en México por Mendoza et al., quienes identificaron familias en rango medio, balanceadas y familias extremas. Al analizar el tipo de comunicación como variable biopsicososial, se detectó predominio de comunicación directa en familias balanceadas, mientras que la comunicación enmascarada se observó en familias de rango medio y la comunicación desplazada en las familias extremas. Finalmente, se concluyó que los más destacados factores relacionados a la funcionalidad fueron la comunicación directa y límites claros (21).

Por su parte, al estudiar el funcionamiento familiar de familias de personas con algún diagnóstico de enfermedad crónica o invalidante, autores $(22,23)$ observaron que los familiares percibían menor cohesión y adaptabilidad familiar. Salgueiro y López en Brasil analizaron el funcionamiento de las familias cuidadoras, relacionando el nivel de dependencia del familiar anciano y la edad de los cuidadores con la dinámica familiar, concluyendo que el nivel de dependencia del anciano no altera la cohesión y adaptabilidad familiar. Sin embargo, la edad avanzada del cuidador afectaría la dinámica familiar, induciendo un potencial patológico (24).

En Chile, con todo lo construido en Salud familiar, aún es incipiente el estudio del funcionamiento de las familias, con personas dependientes de cuidado, en sus dimensiones de comunicación, cohesión y adaptabilidad. Por lo anteriormente expuesto, el objetivo de este estudio fue identificar cómo se relacionan los factores biopsicosociales, factores de riesgo y protectores con el funcionamiento familiar de las familias que tienen un integrante dependiente, desde el punto de vista del paciente y la familia. 


\section{MATERIAL Y MÉTODO}

Diseño transversal, descriptivo, correlacional. Las unidades de análisis fueron el paciente dependiente y su familia (al menos dos familiares). La población correspondió a pacientes y familiares que vivían con un integrante dependiente, adscritos a Centros de Salud Familiar de la ciudad de Chillán. Para la selección de la muestra fueron seleccionados por conveniencia dos centros asistenciales, por encontrarse en un sector territorial cercano y abarcar un número poblacional de características sociales similares.

De acuerdo a la categorización de pacientes dependientes utilizada (leve, moderado y severo), se calculó, mediante estratificación estadística (con un error máximo admisible de $5 \%$ ), el total de familias que debían ser encuestadas, de modo que cada Centro de Salud Familiar tuviese la misma probabilidad de ser elegido, obteniendo 31 familias con un integrante dependiente leve, 71 con un integrante con dependencia moderada y 73 con dependencia severa, conformando una muestra de 175 familias que vivían con un familiar dependiente. Posteriormente, de las 175 familias, 21 de ellas fueron excluidas por: no encontrarse en su domicilio en más de dos visitas, cambio de domicilio o fallecimiento del paciente, quedando conformada la población del estudio por 154 pacientes y 310 familiares.

Se utilizaron los siguientes instrumentos recolectores de datos conformados por:

I) Encuesta biopsicosocial en la que se identificaron las siguientes variables:

- Psicosociales: Nivel educacional del familiar o integrante postrado, ocupación del familiar encuestado, ingreso familiar.

- Variables de estructura familiar: Número de integrantes del núcleo familiar, estructura familiar y etapa del ciclo vital.
- Factores de riesgo familiar: jefe de hogar cesante, historia de alcohol y /o dependencia de drogas de algún miembro de la familia, enfermedades crónicas sin control (hipertensión arterial, diabetes mellitus, enfermedad pulmonar obstructiva crónica, asma, dislipidemia, depresión), violencia intrafamiliar, embarazo adolescente.

- Factores protectores de la familia: Apoyo o pertenencia a instituciones o redes sociales y familiares de apoyo, controles de patologías crónicas al día, del familiar encuestado y Comunicación.

II) Escala FACES III, validada en Chile (15), que evalúa el funcionamiento familiar en las dimensiones, cohesión y adaptabilidad a través de una escala tipo Likert de cinco opciones (casi siempre, muchas veces, a veces sí y a veces no, pocas veces, casi nunca), conformada por 40 ítems, divididos en dos partes. En la primera parte se evalúa el nivel de cohesión y adaptabilidad de la familia tal como el sujeto la percibe en ese momento ("Familia Real”), y en la segunda, lo que a la persona le gustaría que hubiese en su familia ("Familia Ideal”). En este estudio sólo se utilizó la escala "Familia Real" (25).

Según el modelo Circunflejo de Olson, los 16 tipos de sistemas familiares que resultan del cruce entre las dimensiones cohesión (desligada, separada, conectada, enmarañada) y adaptabilidad (caótica, flexible, estructurada y rígida), constituye los siguientes tipos de familias (según puntajes):

1. Puntajes de Cohesión: suma de todos los ítems impares: a.- Para adultos en cualquier estadio del ciclo familiar: 35 puntos o menos: Familia Desligada; 36 puntos a 40: Familia Separada; 41 puntos a 46: Familia Conectada; 47 puntos o más: Familia Enmarañada o Aglutinada

2. Puntajes de Adaptabilidad: a.- Para adultos en cualquier estadio del ciclo familiar: 
19 puntos o menos: Familia Rígida; 20 a 23 puntos: Familia Estructurada; 24 a 29 puntos: Familia Flexible; 30 puntos o más: Familia Caótica.

De estas dimensiones se identifican tres tipos de funcionamiento familiar: Balanceadas, en Rango medio y Extremas.

III) La dimensión comunicación se evaluó con la escala incluida en FACES IV (en su versión en inglés) de Olson (25). Al no tener referencias de la validación de este instrumento en Chile se consideraron los criterios de validación según Hernández et al. (26), en donde las 10 preguntas fueron traducidas del inglés al español por tres expertos y posteriormente se realizó validación facial con 10 personas (adultos y adultos mayores), elegidos al azar para evaluar su comprensión, redacción y consignas sugeridas. Se corrigió nuevamente la redacción de los items en español y se procedió a la evaluación inversa realizada por docente de lengua nativa al inglés. Se realizó una prueba piloto a 26 familias elegidas aleatoriamente en un Centro de Salud Familiar de la ciudad de Chillán, con el propósito de recoger información preliminar en relación a comprensión y entendimiento del instrumento. En el análisis de fiabilidad de la prueba piloto se obtuvo un Alpha de Cronbach de 0.9, lo que muestra una alta consistencia interna de la escala.

Para la recolección de los datos se realizaron entrevistas al paciente y al menos a dos integrantes del grupo familiar. El requisito para entrevistar al familiar fue que viviera más de un año junto al paciente dependiente y que tuviese 17 años o más de edad, previo consentimiento escrito de su voluntad de participar. La información del paciente y familiares se tabuló por separado y los puntajes de cada familiar se promediaron para los instrumentos FACES III y IV.

Se resguardaron los aspectos éticos en el desarrollo de la investigación (27). El proyecto fue evaluado por el Comité de Ética de la
Facultad de Medicina de la Universidad de Concepción y, previa aplicación de los instrumentos recolectores de datos, se solicitó autorización al Departamento de Salud Municipal de Chillán. Se utilizó estadística descriptiva y la prueba de Chi Cuadrado para la asociación entre las variables.

\section{RESULTADOS}

El total de personas entrevistadas fue de 464; de ellos 154 fueron pacientes dependientes y 310 familiares.

\section{Caracterización de los pacientes dependientes y familiares}

El promedio de edad de los pacientes dependientes, correspondió a 64 años; el 56,3\% de ellos de sexo masculino y el $43,7 \%$ de sexo femenino, jubilados en un $68,5 \%$ y con escolaridad básica $71,0 \%$ de los pacientes. Estado civil, casados $(40,1 \%)$ y viudos $(32,1 \%)$. En cuanto a los familiares encuestados, el promedio de edad fue 43 años; predominaron las mujeres $(73,3 \%)$, correspondiendo en la mayoría de los casos a las cuidadoras principales, con un tipo de relación de esposas o hijas; de ocupación actual dueña de casa con un $27,4 \%$ y que además realizan trabajos esporádicos en un $28,0 \%$. El resto corresponde a empleados fiscales y estudiantes. Respecto a la escolaridad, el 39,3\% de los familiares refirieren estudios básicos, seguido por educación media y técnico-profesional. En relación al estado civil, el $41,7 \%$ era soltero, un $40,1 \%$ casado y convivientes un $6,3 \%$.

Las patologías crónicas más frecuentes en pacientes dependientes y familiares correspondieron a las cardiovasculares. En los pacientes prevalecieron patologías cardiovasculares $(44,4 \%)$, seguidas de cáncer, Enfermedad Pulmonar Obstructiva Crónica (EPOC) y artrosis. En los familiares estas 
patologías correspondieron a hipertensión arterial, diabetes, dislipidemia en un $65,4 \%$; seguida por asma, artrosis y EPOC.

En relación a la asistencia a los controles de salud, un $81,5 \%$ de los pacientes cumple con sus controles de salud, mientras que un $55,8 \%$ de los familiares refiere no cumplir con ellos.

\section{Caracterización de las familias}

Respecto de la caracterización de las familias, el ingreso familiar mensual se encontró entre los $\$ 250.000$ y 500.000 mil pesos para un $58 \%$, sin embargo un $42 \%$ de las familias recibe un ingreso inferior entre los $\$ 186.150$ y 250.000 mil pesos. En relación al tipo de familia, se identificó la de tipo extendida monoparental seguida por la nuclear biparental. Un $32,6 \%$ se encontraba en plataforma de lanzamiento y $30,6 \%$ correspondía a familia en edad media.

Los principales factores de riesgo familiares declarados por el paciente y su familiar corresponden a: Cesantía, Alcoholismo, Embarazo no deseado y Violencia Intrafamiliar. De ellos, el menor porcentaje es de: Embarazo no deseado (12,9\%), y el mayor corresponde a Cesantía $(29,8 \%)$. En relación a la percepción de Violencia Intrafamiliar es mayor en los familiares con un $21,9 \%$, y sólo un $11,1 \%$ en los pacientes dependientes.

Los principales factores protectores identificados por el paciente y la familia dependiente corresponden a: la red social. Los pacientes dependientes identifican como red: a sus vecinos $(47,5 \%)$, parientes $(16,0 \%)$ y la Iglesia (10,5\%). Respecto de los familiares, la principal red social referida fueron sus vecinos y parientes con un 42,9 y $26,6 \%$ respectivamente. Referente a la percepción de la comunicación familiar, ésta se identifica como "muy alta" por ambos grupos con un 34,5\% (familiar) y $35,7 \%$ (paciente).

\section{Tipos de funcionalidad familiar}

Al evaluar los tipos de funcionamiento familiar, desde la opinión del paciente dependiente, de los 16 tipos de familias que propone Olson, al combinar los cuatro tipos de Cohesión y cuatro tipos de Adaptabilidad, sólo se encontraron 9 tipos de funcionamiento familiar. El 28\% de los pacientes percibió un funcionamiento familiar conectado-caótico, seguido del 21,4\% separado-caótico, es decir rango medio. Un 19,5\% es extrema, es decir presentan alteración en el funcionamiento familiar. Al respecto, cabe mencionar que solo un pequeño porcentaje es balanceada (Figura 1).

Sin embargo, al analizar la percepción de los familiares encuestados, de los 16 tipos de familias que propone Olson, al combinar los cuatro tipos de Cohesión y cuatro tipos de Adaptabilidad del familiar (Figura 2), sólo se encontraron en las familias estudiadas 12 tipos de ellos. El 24,5\% tuvo un funcionamiento familiar separado-caótico, seguido del 24,0\% conectado-caótico, es decir rango medio. Un $23,9 \%$ es desligada-caótica es decir funcionamiento familiar extremo, presentando alteración en el funcionamiento familiar. Al respecto, tan solo un $5,8 \%$ es balanceado. Desde la perspectiva de los familiares, el nivel de funcionamiento familiar percibido es de rango medio (63,8\%), seguido por tipo de funcionamiento extremo.

\section{Análisis bivariado}

$\mathrm{Al}$ estudiar la relación entre la funcionalidad familiar, según grado de discapacidad, no se encontró asociación entre ambas variables (Tabla 1).

$\mathrm{Al}$ analizar relación entre tipos de comunicación, percibida por el familiar y paciente, y funcionamiento familiar se encontró asociación estadística significativa, sin embargo su nivel de asociatividad fue débil, demostrado por el nivel de Contingencia. 
Figura 1. Tipos de funcionamiento familiar según Modelo de Olson, percepción de los pacientes dependientes encuestados $(n=154)$.

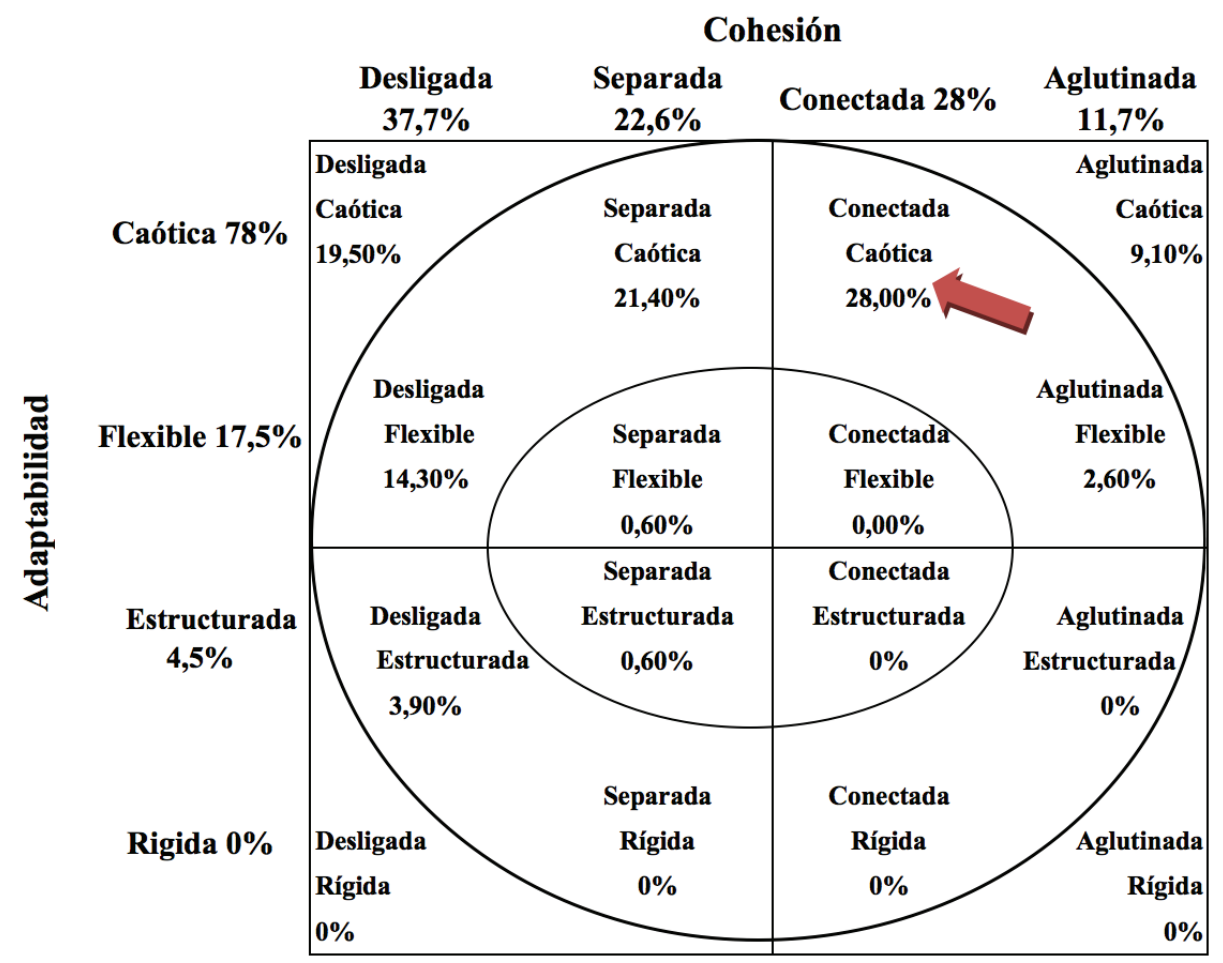

Figura 2. Tipos de funcionamiento familiar según Modelo de Olson, percepción de los familiares encuestados $(n=310)$.

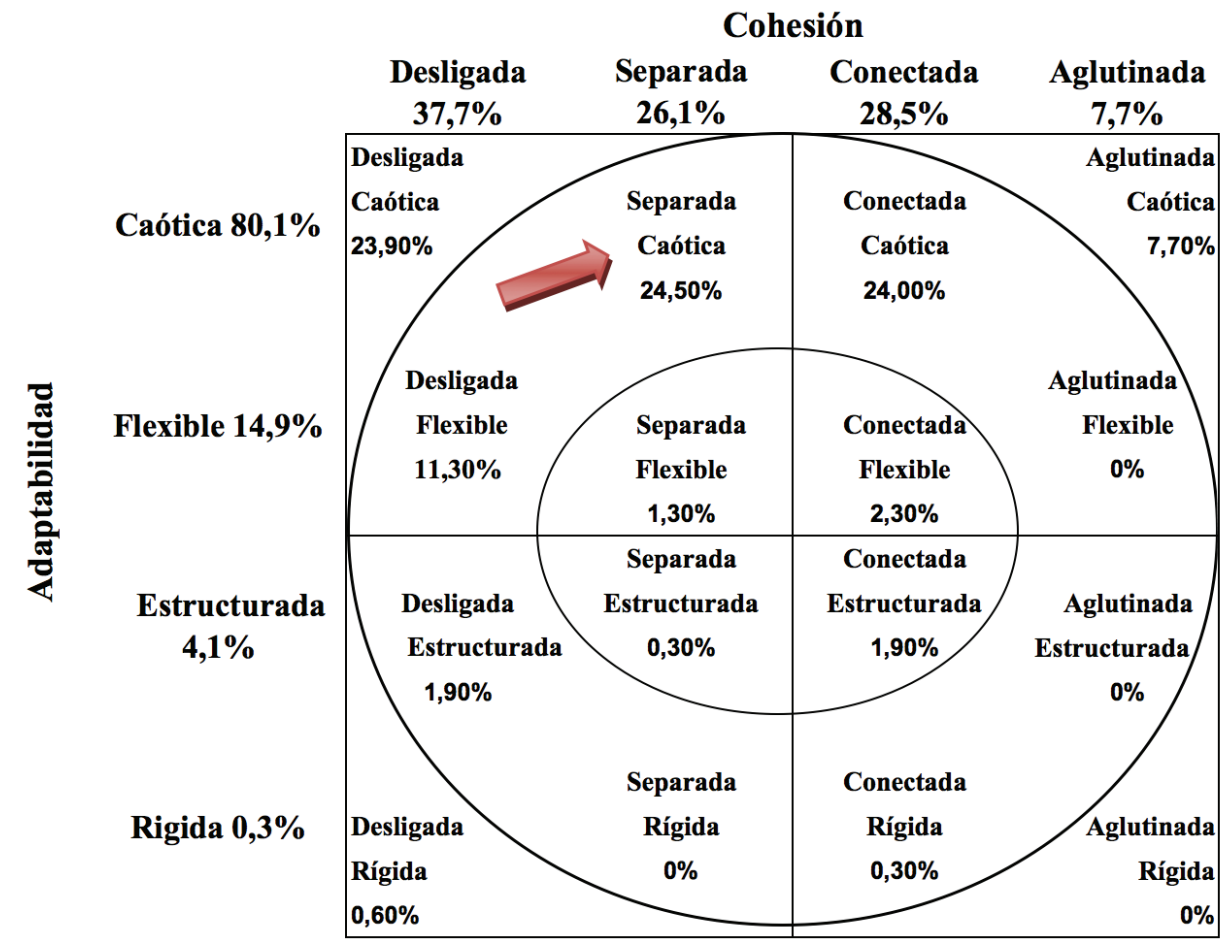


Tabla 1. Distribución de Niveles de Funcionalidad familiar percibidos por el paciente dependiente $(n=154)$ y el familiar $(n=310)$.

\begin{tabular}{|c|c|c|c|c|c|}
\hline & \multicolumn{3}{|c|}{ Nivel de funcionamiento familiar } & \multirow[b]{2}{*}{$\chi^{2}$} & \multirow[b]{2}{*}{$\mathrm{p}$-valor } \\
\hline & $\begin{array}{c}\text { Familia } \\
\text { Equilibrada }\end{array}$ & $\begin{array}{c}\text { Familia Rango } \\
\text { medio }\end{array}$ & $\begin{array}{c}\text { Familia } \\
\text { Extrema }\end{array}$ & & \\
\hline \multicolumn{6}{|l|}{ Grado de discapacidad } \\
\hline \multicolumn{6}{|l|}{ Pacientes } \\
\hline Discapacidad leve & $0(0,0 \%)$ & $32(29,6 \%)$ & $6(13,6 \%)$ & \multirow{5}{*}{7,494} & \multirow{3}{*}{0,112} \\
\hline Discapacidad moderada & $0(0,0 \%)$ & $35(32,4 \%)$ & $19(43,2 \%)$ & & \\
\hline Discapacidad severa & $2(10,0 \%)$ & $41(43,2 \%)$ & $19(38,0 \%)$ & & \\
\hline \multicolumn{5}{|l|}{ Familiar } & \\
\hline Sin discapacidad & $12(4,0 \%)$ & $198(63,8 \%)$ & $100(32,2 \%)$ & & \\
\hline \multicolumn{6}{|l|}{ Tipos de Comunicación } \\
\hline \multicolumn{6}{|l|}{ Paciente $^{*}$} \\
\hline Muy alto & 0 & $43(39,8 \%)$ & $12(27,3 \%)$ & \multirow{5}{*}{16,627} & \multirow{5}{*}{0,034} \\
\hline Alto & 0 & $36(33,3 \%)$ & $17(38,6 \%)$ & & \\
\hline Moderado & $1(50,0 \%)$ & $12(11,1 \%)$ & $11(25,0 \%)$ & & \\
\hline Bajo & $0(0,0 \%)$ & $11(10,2 \%)$ & $2(4,5 \%)$ & & \\
\hline Muy bajo & $1(50,0 \%)$ & $6(5,6 \%)$ & $2(4,5 \%)$ & & \\
\hline \multicolumn{6}{|l|}{ Familiar $^{* *}$} \\
\hline Muy alto & $2(16,7 \%)$ & $77(38,9 \%)$ & $28(28 \%)$ & \multirow{5}{*}{48,265} & \multirow{5}{*}{$<0,001$} \\
\hline Alto & $4(33,3 \%)$ & $72(36,4 \%)$ & $16(16,0 \%)$ & & \\
\hline Moderado & $3(12,6 \%)$ & $25(25 \%)$ & $44(44,0 \%)$ & & \\
\hline Bajo & $3(8,6 \%)$ & $17(25 \%)$ & $12(12,0 \%)$ & & \\
\hline Muy bajo & $0(0,0 \%)$ & $7(3,5 \%)$ & $0(0,0 \%)$ & & \\
\hline
\end{tabular}

Coeficiente de contingencia: ${ }^{\star}=0,312 ;{ }^{* *}=0,367$.

\section{DISCUSIÓN Y CONCLUSIÓN}

Los resultados del estudio revelan que la variable comunicación familiar se relaciona con el tipo de funcionamiento familiar, aunque algunas con relaciones estadísticas débiles. Los familiares que identifican una Comunicación Muy Alto y Alto, presentan un Funcionamiento familiar de rango Medio; mientras que aquellos que identifican un nivel de Comunicación Moderado presenta un funcionamiento familiar de rango Extremo; esto implicaría un funcionamiento familiar más problemático y dificultad para enfrentar eventos estresantes tales como cuidar a un familiar dependiente. No obstante, según los postulados de Olson, se establece que los sistemas no balanceados pueden ser funcionales y los sistemas familiares funcionales pueden ser no balanceados en ciertas ocasiones (28). Los sistemas balanceados pueden comportarse como no balanceados cuando están bajo altos niveles de estrés (29).

Por su parte, el modelo Circumplejo de Olson identifica a la comunicación familiar 
como un tercer elemento facilitador de la dinámica familiar, sin embargo no se grafica en su modelo (13). La investigación confirma este postulado y muestra que las familias y pacientes que perciben una comunicación "muy alta" presentan un funcionamiento familiar más saludable con roles definidos, disciplina y adaptación a cambios frecuentes. Mientras que en el nivel de comunicación moderado y bajo, se identifica con un liderazgo medio, roles ocasionalmente compartidos. Cierto grado de disciplina democrática y cambios sólo cuando son necesarios. Para el familiar que es dueña de casa se presenta una cohesión de tipo conectada, es decir, unión afectiva, fidelidad e interdependencia entre sus miembros; para el familiar que es trabajador esporádico, cohesión de tipo desligada, es decir ausencia de unión afectiva y lealtad. Para el familiar estudiante se presenta una cohesión de tipo enmarañada, es decir una alta dependencia familiar en la toma de decisiones.

Aun cuando no se encontró relación entre el grado de dependencia del paciente y el tipo de funcionamiento familiar, cabe mencionar que el paciente dependiente $(1,2 \%)$ y el familiar $(5,8 \%)$ perciben un funcionamiento familiar balanceado en un porcentaje muy inferior; esto podría relacionarse a la presencia de diferentes factores de riesgo entre ellos: vivir y cuidar un integrante dependiente o presentar otras variables de riesgo tales como violencia intrafamiliar, embarazo no deseado y alcoholismo. Por su parte, los familiares presentan un nivel de funcionamiento familiar de rango medio (63,8\%), esto conllevaría a un funcionamiento familiar más saludable; sin embargo de acuerdo a la hipótesis de Olson, estas familias podrían pasar de un estado de funcionalidad a disfuncionalidad extrema, debido a otros factores de riesgo o vulnerabilidad que afectarían la comunicación familiar. Por lo anterior, detectar grupos o familias vulnerables de presentar problemas de salud o alteración en su funcionamiento, con la generación de un sistema de evalua- ción válido y multidimensional, permitiría reflejar la complejidad de los factores que se van a intervenir, logrando mayor impacto en la protección de dicho núcleo, optimizando de este modo los recursos disponibles.

Cabe señalar que los resultados permitieron dar respuesta al objetivo de la investigación, concluyendo que la comunicación como factor protector es un tercer elemento fuertemente asociado a la dinámica familiar. Estos resultados reafirman lo planteado por Mendoza, et al. (21), quienes concluyen que los más destacados factores relacionados al funcionamiento familiar son: la comunicación directa y límites claros.

Bajo la visión de enfermería ha existido un avance en torno al cuidado de la familia, pero son escasos los estudios o publicaciones que profundicen en el funcionamiento familiar; a su vez los instrumentos para diagnóstico familiar son diversos y abarcan las dimensiones de la persona y su familia en forma generalizada.

Como limitaciones de este estudio es posible mencionar que no siempre las personas y sus familiares presentaron disponibilidad a ser entrevistadas, y también las direcciones de los hogares que ellos informaron en sus Centros de Salud no correspondieron a su domicilio; quizás por un desconocimiento de los derechos y deberes de las personas.

Las conclusiones de esta investigación proveen una base para futuros estudios que puedan contribuir a investigar a las familias en nuestro país, constituyendo un importante aporte; siendo una herramienta práctica para la disciplina de enfermería y otras áreas de la salud que trabajan en Atención Primaria, ya que nos permiten mostrar evidencia científica para el diseño y continuidad de programas de salud familiar con la valoración de riesgos por grupos de edad, biológico-familiares del medio ambiente, de prácticas y de servicios de salud; permitiendo de esta manera planificar acciones anticipatorias para disminuir riesgos y evitar una disfuncionalidad familiar. 


\section{REFERENCIAS}

1. Luengo C, Araneda G, López MA. Factores del cuidador familiar que influyen en el cumplimiento de los cuidados básicos del usuario postrado. Index Enferm. 2010; 19(1): 14-18.

2. Irarrázaval M, Prieto F, Armijo J. Prevención e intervenciones tempranas en salud mental: una perspectiva internacional. Acta bioeth. 2016; 22(1): 37-50.

3. Pizarro M, Espinosa F, Muñoz L, Peralta E, Laferte M. Análisis descriptivo en cuidadores de personas postradas del consultorio Pedro Pulgar, comuna de Alto Hospicio. Rev. Soc. Med. 2008; III(3): 864-869.

4. Espinoza K, Jofre V. Sobrecarga, apoyo social y autocuidado en cuidadores informales. Cienc. enferm. [Internet]. 2012 Ago [citado 08 mar 2016]; XVIII (2): 23-30. Disponible en: https://scielo. conicyt.cl/scielo.php?script=sci_arttext\&pid=S0717-95532012000200003

5. McDaniel S. Orientación Familiar en Atención Primaria. 2a ed. Barcelona: Springer Science \& Business Media; 2000. $432 \mathrm{p}$.

6. Depaux R, Campodónico L, Ringeling I, Segovia I. En el camino a centro de Salud Familiar [Internet]. Chile: Subsecretaría de Redes Asistenciales, Ministerio de Salud (Chile); 2008 [citado 03 marzo 2016]. 132 p. Disponible en: http://www. bibliotecaminsal.cl/wp/wp-content/ uploads/2016/03/En-el-Camino-a-Centro-de-Salud-Familiar.pdf

7. Subsecretaría de Redes Asistenciales, División de Atención Primaria, Departamento de Diseño y Gestión de APS. Manual de Apoyo a la Implementación del Modelo de Atención Integral con Enfoque Familiar y Comunitario en Establecimientos de Atención Primaria [Internet]. Santiago, Chile: Subsecretaría de Redes Asistenciales (CL); 2008 [citado 03 mar
2016]. 191 p. Disponible en: http://www. odontoredes.com/2013/06/modelo.html

8. Código Sanitario. Decreto con Fuerza de Ley $N^{\circ} 725$, Libro V, Artículo 113, inciso cuarto, modificado por Ley 19536, Art 7 a), que concede una bonificación extraordinaria para enfermeras y matronas que se desempeñan en condiciones que indica, en los establecimientos de los Servicios de Salud. D.O.16.12.1997. Disponible en: http://www.leychile.cl/Navegar?idNorma $=81502$

9. Hidalgo C. Carrasco E. Salud familiar. Un modelo de atención Integral en la Atención Primaria. 2a ed. Santiago, Chile: Edic. Universidad Católica de Chile; 2002. 438 p.

10. Olson D, Gorall D. Circumplex model of marital and family systems. En: Walsh F (ed). Normal family processes: Growing diversity and complexity. 3rd ed. New York: Guilford; 2003. p. 514-548.

11. Friedemann ML, Chávez M, Alcorta A. Evaluación de la escala de efectividad de la funcionalidad familiar. Desar Cien de Enf. 2000; 8(1): 12-18.

12. Martínez A, Iraurgi I, Galíndez E, Sanz M. Family Adaptability and Cohesion Evaluation Scale (FACES): desarrollo de una versión de 20 ítems en español. Int J Clin Health Psychol. 2006; 6(2): 317-338.

13. Olson DH. Circumplex Model of Marital and Family Systems. J Fam Ther. 2000; 22(2): 144-167.

14. Olson DH. FACES IV and the Circumplex model: Validation study. J Marital FamTher. 2011; 37(1): 64-80.

15. Zegers B, Larraín M, Polaino-Lorente A, Trapp A, Diez I. Validez y confiabilidad de la versión española de la escala de Cohesión y Adaptabilidad Familiar (CAF) de Olson, Russell \& Sprenkle para el diagnóstico del funcionamiento familiar en la población chilena. Rev Chil Neuropsiquiatr. 2003; 41(1): 39-54.

16. Vargas J, González C. Escuela para padres: el funcionamiento familiar. Centro 
Regional de Investigación en Psicología. 2009; 3(1): 19-22.

17. González I. Las crisis familiares. Rev Cubana Med Gen Integr [Internet]. 2000 Jun [citado 05 sep 2013]; 16(3): 270-276. Disponible en: http://scielo.sld.cu/scielo.php?script=sci_ arttext\&pid=S0864-21252000000300010

18. Espinoza E, Méndez V, Lara R, Rivera P. Factores asociados al nivel de sobrecarga de los cuidadores informales de adultos mayores dependientes, en control en el consultorio José Durán Trujillo, San Carlos, Chile. Theoria. 2009; 18(1): 69-79.

19. Schmidt V, Barreyro JP, Maglio AL. Escala de evaluación del funcionamiento familiar FACES III: ¿ Modelo de dos o tres factores? Escr Psicol. 2010; 3(2): 30-36.

20. Ferrer P, Miscán A, Pino M, Pérez V. Funcionamiento familiar según el modelo complejo de Olson en familias con un niño que presenta retardo mental. Rev enferm Herediana. 2013; 6(2): 51-58.

21. Mendoza LA, Soler E, Sainz L, Gil I, Mendoza HF, Pérez C. Análisis de la Dinámica y Funcionalidad Familiar en Atención Primaria. Archivos en Medicina familiar. 2006; 8(1): 27-32.

22. Maglio AL, Molina MF. La familia de adolescentes con trastornos alimentarios ¿Cómo perciben sus miembros el funcionamiento familiar? Rev Mex Trastor Aliment [Internet]. 2012 Jun [citado 15 nov 2014]; 3(1): 1-10. Disponible en: http:// www.scielo.org.mx/scielo.php?script $=$ sci_ arttext\&pid=S2007-15232012000100001
23. Ornelas J, Sánchez O. Cohesión y adaptabilidad familiar en mujeres con Epilepsia: estudio transversal analítico. Aten Primaria. 2014; 46(5): 246-253.

24. Salgueiro H, Lopes M. A dinâmica da família que coabita e cuida de um idoso dependente. Rev Gaucha Enferm. 2010; 31(1): 26-32.

25. Costa D, González M, Masjuan N, Trápaga M, Del Arca D, Scafarelli L, et al. Escala de evaluación del funcionamiento familiar -FACES IV-: Proceso de adaptación a Montevideo, Uruguay. Ciencias Psicológicas. 2009; III(1): 43-56.

26. Hernández R, Fernández C, Baptista $\mathrm{P}$. Metodología de la investigación. $3^{a}$ ed. México: Mc Graw-Hill-Interamericana; $2003.705 \mathrm{p}$.

27. Emanuel E. ¿Qué hace que la Investigación Clínica sea Ética? Siete Requisitos Éticos. En: Lolas F, Quezada A, editores. Pautas éticas de investigación en sujetos humanos: nuevas perspectivas. Santiago: Programa Regional de Bioética OPS/ OMS; 2003. p. 83-95.

28. Olson DH, Russell CS, Sprenkle DH. Circumplex model of marital and family systems: VI. Theoretical update. Fam Process. 1983; 22(1): 69-83.

29. Ponce ER, Gómez FJ, Terán M, Irigoyen AE, Landgrave S. Validez de constructo del cuestionario FACES III en español (México). Aten Primaria. 2002; 30(10): 624-630. 